Société d'histoire de la révolution de 1848 et des

révolutions du XIXe siècle

29 | 2004

Varia

\title{
Une «barricade de papiers » : le pétitionnement contre la restriction du suffrage universel masculin en mai 1850
}

François Jarrige

\section{OpenEdition}

Journals

Édition électronique

URL : http://journals.openedition.org/rh19/698

DOI : $10.4000 /$ rh 19.698

ISSN : $1777-5329$

\section{Éditeur}

La Société de 1848

Édition imprimée

Date de publication : 1 décembre 2004

Pagination : 53-70

ISSN : 1265-1354

\section{Référence électronique}

François Jarrige, "Une « barricade de papiers » : le pétitionnement contre la restriction du suffrage universel masculin en mai 1850 », Revue d'histoire du XIXe siècle [En ligne], 29 | 2004, mis en ligne le 29 juin 2005, consulté le 19 avril 2019. URL : http://journals.openedition.org/rh19/698 ; DOI : 10.4000/ rh19.698

Ce document a été généré automatiquement le 19 avril 2019

Tous droits réservés 


\title{
Une « barricade de papiers »: le pétitionnement contre la restriction du suffrage universel masculin en mai 1850
}

\author{
François Jarrige
}

« Pétitions! Pétitions! Pétitions ! Que ce cri retentisse en échos formidables d'un bout de la France à l'autre, qu'il éclate comme la foudre à chaque rue, sous chaque toit de la capitale, qu'il se

répande dans les âmes et se traduise en une activité militante, énergique, incessante. Que les pétitions se signent à toute heure et partout, aux

bureaux de la presse Républicaine, dans les magasins, dans les ateliers, au foyer domestique.

Que les hommes de cœur et de dévouement y

consacrent tous les efforts de leur zèle, toute

l'énergie de leurs convictions. Le temps presse ; un jour, une heure, un moment perdus sont un crime. Debout tout ce qui a du feu dans la poitrine, de la force dans l'âme! Debout tout ce qui veut la Constitution, tout ce qui est républicain, tout ce qui est peuple! Debout Paris et les départements! Debout la France entière ! Que chaque nom soit pavé, chaque signature un fusil, chaque pétition une barricade, et nous verrons quelle armée pourra vaincre cette Révolution du pétitionnement. » La Voix du Peuple, 14 mai 1850 
$1 \quad$ Malgré les résultats modérés des premiers scrutins au suffrage universel en 1848, les conservateurs de la Seconde République ont rapidement cherché à canaliser l'exercice du droit de vote en lui imposant diverses limites. Jusqu'à la fin de 1849, toutefois, il s'agit davantage de « circonvenir » le suffrage que de le remettre en cause de manière radicale. À partir de 1850, les attaques se renforcent. Le 22 janvier 1850, le journal légitimiste L'Assemblée nationale présente ainsi un projet de réforme électorale extrémiste visant à substituer au suffrage universel direct un scrutin à plusieurs degrés ${ }^{1}$. Les craintes conservatrices s'exacerbent après les élections partielles du 10 mars 1850 qui voient la victoire symbolique de Hippolyte Carnot, puis le succès du romancier socialisant Eugène Sue à Paris le 28 avril 1850. Dans ce contexte tendu, une commission extraparlementaire composée de dix-sept notables légitimistes et orléanistes est créée en vue de préparer une nouvelle loi électorale. Le 31 mai 1850 la majorité de l'Assemblée vote ce projet de loi que Marx décrira, en raison de ses conséquences décisives sur le destin de la Seconde République, comme le « premier coup d'État de la bourgeoisie » ${ }^{2}$.

Ce vote marque indéniablement un tournant dans l'évolution conservatrice du régime. En portant la durée de domicile exigée de six mois à trois ans, et en étendant le champ des incapacités, la loi du 31 mai, qui ôte le droit de vote à près de trois millions d'électeurs (leur nombre passant environ de 9600000 à 6800 000), supprime de fait le suffrage universel masculin. Les nouvelles dispositions de la loi électorale frappaient d'abord les ouvriers itinérants (compagnons faisant leur tour de France, ouvriers chassés par la crise, migrants de toutes sortes) ${ }^{3}$. À travers cette loi, c'était aussi la capitale qu'on avait voulu frapper; et c'est bien à Paris et dans le département de la Seine que la proportion d'électeurs radiés fut la plus importante $(56,57 \% \text { et } 57,18 \%)^{4}$. La loi électorale marqua également un tournant dans l'évolution du régime, comme le note Maurice Agulhon: "tout le jeu politique fut changé ${ }^{5}$. Si les étapes de la promulgation de cette loi sont désormais bien connues, en revanche on a moins étudié les réactions qu'elle suscita dans le pays. Celles-ci furent pourtant remarquables à plus d'un titre.

3 Dès l'annonce du projet de loi, les organes de l'opposition protestent et le pétitionnement s'impose rapidement comme le mode d'action privilégié. En quelques jours, les journaux républicains comme les clubs et comités démocratiques mettent sur pied une vaste campagne de pétitions qui, en seulement trois semaines, recueille environ 500000 signatures sur plus de 7000 pétitions collectées dans l'ensemble du pays. Malgré son ampleur, cette campagne n'a jamais été étudiée en détail par l'historiographie, qui se contente le plus souvent de la mentionner rapidement, voire de façon erronée ${ }^{6}$. Pourtant, elle constitue à la fois une étape déterminante de l'expérience politique républicaine et la plus importante mobilisation de l'opinion en France dans la première moitié du XIX ${ }^{e}$ siècle ${ }^{7}$. En outre, l'étude de ces pétitions, qui peut être menée à la lumière des récents travaux de la sociologie politique ${ }^{8}$, s'avère particulièrement riche pour saisir les singularités des pratiques et des représentations politiques d'une époque dominée par l'apparente passivité des masses face au renforcement de la répression et du contrôle social. Contre une vision téléologique de la Seconde République, réduite à deux moments fondateurs (la Révolution de Février et le coup d'état du 2 décembre), la prise en compte de cette campagne d'opinion invite au contraire à rechercher les espaces où se replie l'activité démocratique. Dans cette perspective, nous procéderons à une déconstruction de cette campagne de pétitions en examinant les différentes phases par lesquelles passent les textes de protestation. On suivra d'abord l'élaboration et les conditions matérielles de collecte des pétitions. Puis, on s'attachera à cerner les principales caractéristiques de 
cette campagne en étudiant les singularités de la parole et de la géographie pétitionnaire. Enfin, en suivant les pétitions du village au Parlement, nous questionnerons les usages pluriels de ces textes de protestation dans l'arène politique.

La « Révolution du pétitionnement »

4 Une fois connu, le projet des Burgraves suscite immédiatement l'indignation et la protestation unanimes des démocrates. Rapidement, la discussion s'engage sur les modes d'action à mettre en œuvre pour s'opposer au projet de loi. Fidèle à ce que Maurice Agulhon appelle "l'orientation légaliste» des chefs du parti rouge, les républicains rejettent la voie insurrectionnelle au profit d'une protestation pacifique ${ }^{9}$. Dès le 7 mai, $L a$ Presse de Girardin appelle l'ensemble des journaux républicains à se positionner sur cette question. Le lendemain, $L a$ Démocratie Pacifique lui répond par un véritable programme d'action : «Cet ajournement des moyens extrêmes [...] est devenu, pour la démocratie au dix-neuvième siècle, un devoir d'humanité, un titre d'honneur, une preuve de supériorité et de progrès, un moyen même des plus habiles, des plus sûrs, de convertir aux idées nouvelles les âmes honnêtes, les hommes de cœur qui, quels que soient leurs anciens partis, se voient entraîner à rendre hommage à la modération des opprimés et à condamner les oppresseurs ». En conséquence, poursuit le rédacteur du texte, « il y aura d'abord à protester par des pétitions couvertes de millions de signatures contre la violation commise ». Malgré les sarcasmes de Marx, pour qui les démocrates français " essayèrent soudain de faire agir le pays à leur place [...] au moyen des pétitions " ${ }^{10}$, ce choix apparaissait logique aux montagnards qui attendaient beaucoup de la propagande pacifique et des élections prévues en 1852 pour reconquérir le pouvoir.

La résistance démocratique prend donc la forme du pétitionnement. Compte tenu du peu de temps disponible pour organiser la résistance, la presse met en place une série de stratégies d'optimisation de la collecte. Au cours de cette période d'élaboration de la forme partisane, la presse démocratique constitue, en effet, le principal relais de la mobilisation politique. Ce souci d'efficacité se retrouve d'abord dans la forme même des pétitions : les journaux publient dans leurs colonnes des "pétitions affiches ${ }^{11}$ dont la fonction première est d'instaurer un véritable barrage du nombre. Ces textes sont souvent accompagnés d'un mode d'emploi à l'usage des militants locaux. En Dordogne, par exemple, La Nouvelle Ruche accompagne sa pétition d'un «avis » qui précise que " chacun [des] abonnés doit détacher cette pétition du journal, la signer et faire signer par tous ses amis, puis la plier en forme de lettre, l'affranchir et la mettre à la poste dans l'espace de vingt quatre heures ${ }^{12}$. Au sein de la presse parisienne, le journal de Proudhon La Voix du peuple est le premier à publier un texte de pétition dans ses colonnes le 12 mai 1850, mais le journal est interdit dès le 14 mai, et la pétition n'a pas le temps de se répandre en province. La Démocratie pacifique, Le National et Le Siècle publient en même temps un texte identique, rédigé par les députés républicains de la réunion Goudchaux (Dupont de l'Eure, David d'Angers, Marrast) ${ }^{13}$. Le même jour, La Presse, la feuille d'Émile de Girardin, publie un texte différent qui va jouer un rôle capital dans la réussite du pétitionnement. Ce texte bref, se prêtant parfaitement à une collecte rapide, est recopié dans presque tous les départements.

Une fois les textes établis et publiés, il s'agit de leur donner la publicité la plus large et de les diffuser dans l'ensemble du territoire. Comme le souligne Léon Faucher lui-même lors du rapport qu'il fait à l'Assemblée ${ }^{14}$, « une pétition copiée sur le modèle fourni par M. Émile de Girardin, et que son journal a publiée, a été présentée dans tous les lieux publics aux citoyens les moins éclairés [...] Dans l'arrondissement de Béziers, des protestations 
contre le projet ont été colportées de cabaret en cabaret, dans les cafés, sur les places publiques, partout où se réunissent les oisifs et les citoyens tarés $"{ }^{15}$. Les rapports des procureurs généraux nous fournissent de précieuses indications sur les lieux et les modalités de la collecte. Les pétitions sont signées dans les espaces traditionnels de la sociabilité populaire. En ville, on signe d'abord dans les « Parlements du Peuple » que sont les cabarets ${ }^{16}$. Les aubergistes et débitants de boisson jouent un rôle crucial de propagandistes de la cause démocratique, à l'image du citoyen Leleux, aubergiste à Assissur-Serre (Aisne), condamné à quatre mois d'emprisonnement pour avoir fait signer une pétition. Dans son rapport, le procureur général note que « Leleux, qui est connu pour ses opinions exaltées, avait copié une pétition demandant le rejet de la loi tendant à réglementer le suffrage, il l'avait placée dans son auberge sur une table avec une pétition relative au crédit foncier et il incitait tous ceux qui venaient chez lui à les signer, mais il ne se contentait pas simplement de les engager à apposer leur signature, réunissant autour de lui des gens qui partageaient ses opinions, il usait à l'aide de cet entourage et d'un langage menaçant d'une espèce de violence morale pour contraindre ceux auxquels il s'adressait à suivre ses conseils » ${ }^{17}$. Par delà le caractère normatif inhérent à ce type de source, on peut y lire en creux les formes concrètes de la mobilisation. La typologie des lieux de la collecte révèle l'extraordinaire foisonnement des espaces politiques populaires: les réunions des sociétés de secours mutuels, les marchés, la sortie de la messe sont autant d'occasion de mobiliser l'opinion ${ }^{18}$. Les lieux de travail ne sont pas oubliés, et les colporteurs courent les rues, les ateliers et les champs pour recueillir le plus grand nombre de signatures ${ }^{19}$. À Paris, les journaux républicains organisent la signature de leur pétition au siège du journal lui-même : La Presse du 15 mai précise ainsi qu'« à l'heure où nous écrivons, dix heures du soir, l'affluence n'a pas encore diminué dans nos bureaux ${ }^{20}$.

7 Grâce à la diversité de ces modes de collectes, le pétitionnement contre la restriction du suffrage universel est devenu une étape majeure dans la démocratisation de la pratique pétitionnaire. L'origine populaire des pétitionnaires apparaît d'abord dans les silences de la sociologie: on remarque en effet un gonflement du nombre des inconnus. Le prestige des notables et des paroles qualifiées perd de son importance par rapport aux pétitionnements de la période de la monarchie parlementaire ${ }^{21}$. Toutefois, l'effet de cette démocratisation est ambivalent puisque les pétitionnaires semblent perdre en légitimité ce qu'ils ont gagné en effectifs. Dans la forme et l'expression des revendications, ils vont s'efforcer de compenser ce déficit de légitimité en procédant à une reconstruction symbolique du champ du pouvoir.

Émanciper la parole pétitionnaire

8 Le poids du nombre semble en effet insuffisant pour faire plier la majorité conservatrice "frappée de stupeur» devant l'ampleur de la mobilisation ${ }^{22}$. L'argumentation pétitionnaire va compléter cette stratégie du nombre en l'accompagnant de diverses stratégies de captation de légitimité. Pour être efficace, la protestation doit en effet parvenir à rompre les hiérarchies reconnues et les codes de l'adresse qui enchaînent en général la rhétorique pétitionnaire aux contraintes de la déférence politique. L'étude des discours révèle ainsi une série de stratégies rhétoriques visant à reconstruire le champ du pouvoir au profit des pétitionnaires ${ }^{23}$.

9 En premier lieu, comme le révèle l'usage du mot "peuple », les pétitions suivent assez fidèlement la rhétorique républicaine quarante-huitarde. On observe en effet une surabondance du mot «peuple » (57 occurrences) par rapport aux autres termes qui lui 
servent de pendant dans le discours typiquement ouvrier (« ouvriers » et «travailleurs » n'apparaissent qu'à cinq reprises). Par son contour flou, ce terme permet d'englober la totalité sociale dans une même protestation. En supprimant les divisions de classes et les conflits sociaux, il permet de récuser les arguments avancés par le parti de l'ordre pour soutenir le projet de loi (notamment le spectre de l'anarchie et des «classes dangereuses ») ${ }^{24}$. Mais c'est le recours incessant et quasi obsessionnel au «droit » (98 occurrences) et à la constitution (65 occurrences avec ses dérivés comme «constitutionnel») qui rend sans doute le mieux compte de la singularité de la protestation pétitionnaire. Grâce à ces notions clés, les pétitionnaires élaborent une argumentation d'autorité seule à même de libérer le locuteur de sa position d'infériorité vis-à-vis des parlementaires conservateurs ${ }^{25}$. En affirmant que «la constitution est audessus des pouvoirs publics qui n'ont d'existence que par elle " ${ }^{26}$, en répétant l'article 110 qui souligne que «L'Assemblée nationale confie le dépôt de la présente constitution et des droits qu'elle consacre à la garde et au patriotisme de tous les Français » ${ }^{27}$, les pétitionnaires se positionnent résolument du côté du droit et de la légalité contre un pouvoir repoussé dans le champ de l'illégalité.

10 Malgré leur brièveté, ces textes développent donc une argumentation subtile qui repose, en second lieu, sur l'instrumentalisation des mots et des thèmes classiques du camp conservateur. Ainsi les promoteurs du projet de loi deviennent-ils « la faction des $17 »{ }^{28}$, la «faction royaliste»; on dénonce leur immoralité en soulignant que ce sont "des hommes égarés par l'égoïsme» ${ }^{29}$. Or, le mot "faction», hérité de la Révolution, et l'accusation d'immoralité sont des thèmes traditionnels de l'argumentaire anti-socialiste ${ }^{30}$. Plus globalement, les pétitionnaires retournent l'argumentation réactionnaire contre elle-même en lui empruntant certains de ses principaux traits. Ainsi la thèse de la mise en péril, qui constitue l'une des formes majeures de la rhétorique réactionnaire ${ }^{31}$, se retrouve-t-elle fréquemment dans les discours pétitionnaires. Les habitants de Pontoise, par exemple, demandent le rejet d'un " projet de loi dont le premier tort est d'avoir fait naître une agitation fatale à tous les intérêts et de nature à troubler le repos public ». Les pétitionnaires sont conscients des implications de la nouvelle loi, qui instaure «la capacité domiciliaire », selon l'heureuse expression de Cavaignac ${ }^{32}$ : en faisant dépendre l'inscription sur les listes électorales d'une clause de domicile, elle met les ouvriers sous la dépendance des patrons. La loi exacerbe donc les luttes de classes au lieu de les supprimer, elle fait naître des germes nouveaux d'antagonismes entre le capital et le travail. Pour les pétitionnaires, l'instauration du suffrage universel a permis de pacifier le champ politique en apprenant aux masses le sens de la légalité. Contre le parti de l'ordre qui y voit une source de désordre, ils insistent au contraire sur la fonction pacificatrice du suffrage universel. Les pétitionnaires poursuivent l'utopie quarante-huitarde d'un "sacrement de l'unité sociale" par l'exercice du droit de vote ${ }^{33}$. Derrière ce retournement, il y a à la fois une part d'ironie envers les arguments simplistes qu'on opposait au suffrage universel, et une part de menace à destination de tous ceux qui prétendent s'opposer à la République.

11 La menace apparaît également dans la manière de s'adresser aux autorités politiques, et notamment dans l'usage des termes "mandataire» et "mandat» par lesquels les pétitionnaires opèrent une subtile captation de légitimité. L'emploi abondant de ces notions politiques vise à libérer les pétitions de la domination parlementaire en reconstruisant symboliquement la hiérarchie du pouvoir. Les pétitionnaires rappellent sans cesse qu'ils sont à l'origine du pouvoir des représentants, car ce sont eux qui ont 
délivré le mandat: «Non, la majorité [...] ne voudra pas déchirer son mandat, car la déchéance du mandant entraîne celle du mandataire ${ }^{34}$. La pétition Girardin - celle qui est diffusée par La Presse - affirme que « le mandataire qui anéantit le droit du mandant détruit son mandat : Voilà le principe, tirez en la conséquence ». De nombreuses pétitions reprennent comme une litanie cette formule : «le mandataire qui anéantit le droit du mandant se détruit lui-même ». Les pétitionnaires quittent l'habit d'aimables demandeurs pour revêtir celui de l'opposant politique. Ils exigent, en rappelant qu'ils sont les maitres, que l'Assemblée leur obéisse. Par ailleurs, ce recours aux termes "mandat» et «mandataire » plutôt qu'à celui de "représentant» s'inscrit dans un renversement de portée philosophique plus large. Dans la philosophie politique de la représentation démocratique la notion de mandataire s'oppose en effet à celle de représentant. Le représentant est «l'outil d'une véritable institution du politique. La figure du députéorgane qui s'élabore dans ce cadre est opposée à celle du mandataire traditionnel. Il y a en effet toujours une ou des personnes physiques derrière le mandataire alors qu'il n'y a qu'une personne virtuelle derrière l'organe ». Sieyès faisait la distinction entre les « simples porteurs de vote (les mandataires) et les vrais représentants »: «les premiers sont des intermédiaires mécaniques alors que les seconds produisent quelque chose qui ne pouvait se manifester avant leur intervention: la volonté commune ${ }^{35}$. Les pétitionnaires se livrent donc à une véritable reconstruction des bases théoriques de la représentation démocratique. Ils reviennent à la distinction de Sieyès en faisant des membres de l'assemblée législative de simples porteurs mécaniques d'un pouvoir qui leur est supérieur et que les pétitionnaires prétendent détenir. Les pétitionnaires recourent donc à une distinction classique de la philosophie politique pour reconstruire le champ du pouvoir.

12 Jusqu'ici nous avons considéré la protestation pétitionnaire comme un ensemble homogène. Il existe pourtant des lignes de fractures et des niveaux divergents dans les discours, qui apparaissent notamment dans les singularités de la protestation des femmes. Simples intermédiaires de la misère sociale et des doléances domestiques, la voix des femmes est peu présente dans les pétitionnements politiques ${ }^{36}$. Pourtant, à l'occasion de cette campagne, près de 900 femmes signent des pétitions entièrement féminines ${ }^{37}$. Au moyen d'une rhétorique subtile, elles instrumentalisent les représentations traditionnelles des rôles sociaux féminins pour dénoncer le projet de loi. À l'écart des débats politiques, rejetées du droit de suffrage en 1848, ces femmes protestataires élaborent une parole singulière qui mêle la religiosité, l'appel à la morale et à la sphère domestique. Elles ne se placent pas sous la protection du droit entériné par la constitution; le mot même de "constitution » est absent de leur texte alors qu'il forme l'armature des pétitions masculines. Quand elles évoquent le droit, il s'agit du « droit que Dieu a donné [au travailleur] en naissant ${ }^{38}$. Elles demandent aux représentants de ne se "laisser guider que par des sentiments religieux ». Toute une religiosité romantique et saint-simonienne irrigue ces textes de femmes : « Nouvelle Babylone nous courrons après des ruines, pendant qu'animés de l'amour divin nous pourrions encore, nous inspirant de cet amour, ne former qu'une même famille unie par les liens de la solidarité ${ }^{39}$.

Cette religiosité conduit elle-même à une certaine vision de l'harmonie sociale. Dans ces textes, les conflits tendent à s'atténuer, et les femmes en appellent à une pacification des rapports sociaux. Elles demandent aux représentants « de déposer dans l'urne un vote de paix et de conciliation», elles pétitionnent pour imposer «le silence aux passions politiques ». La valorisation de «l'humanité » en général, la référence aux hommes dans 
leur universalité, permettent d'évacuer les sordides luttes politiques des partis. L'instauration de l'harmonie dans les rapports humains est précisément l'un des grands objectifs poursuivis par les saint-simoniens ${ }^{40}$. Cette valorisation de l'harmonie, cet appel à la paix et ce refus de la violence correspondent bien au nouveau style de vie politique que Geneviève Fraisse a entrevu dans les textes des "femmes libres de 1848 " ${ }^{41}$. En se plaçant à l'écart des conflits masculins, les femmes démocrates soulignent la spécificité de leur fonction dans le champ politique. Elles défendent la paix et les mœurs pacifiques face à la violence brutale des hommes. Elles revendiquent ainsi indirectement un rôle dans l'arène politique: apaiser les tensions et résorber les conflits. D'ailleurs, elles utilisent abondamment le vocabulaire moral («sentiment-ressentiment », « la haine et le cœur ») : «le génie du mal triomphera t-il donc toujours ?» demandent-elles. Mais, comme le précise Geneviève Fraisse, ce moralisme doit être distingué de la moralisation des femmes jugée nécessaire au progrès social par les philanthropes ${ }^{42}$. Le moralisme des femmes est un système d'argumentation qui devait permettre d'assurer l'avènement des droits en mettant en avant les devoirs. Le moralisme des femmes de 1848, de même que l'insistance sur leur rôle de mère, est « une tactique de réassurance à l'égard du monde masculin et de son pouvoir " ${ }^{43}$. C'est aussi " au nom de leurs enfants ", " comme épouses et comme mères", qu'elles protestent ${ }^{44}$. Même dans la protestation politique, l'autonomie de la femme ne saurait être complète. À travers leurs plaintes, les femmes ne révolutionnent donc pas les rapports de sexe et de pouvoir; pacifisme et moralisme structurent leur argumentation. Cette rhétorique spécifique ne doit pourtant pas être analysée comme une plate confirmation de la religiosité des femmes du XIXe siècle : c'est avant tout une conséquence de leur position politique ambiguë ${ }^{45}$. Le vocabulaire et les particularités de la protestation rendent compte de la singularité de leur position. Comment, en effet, revendiquer le suffrage universel au nom du droit alors que l'on est soi-même exclu de ce même suffrage dit « universel » au nom du droit? La famille, la religion et la morale deviennent autant de palliatifs d'un droit dont elles sont privées. La femme se fait la protectrice du suffrage universel masculin, elle prend la posture de l'arbitre extérieur (d'où l'appel à Dieu et à la morale), elle tente d'imposer le «silence aux passions politiques» en appelant à une réconciliation entre les hommes du parti de l'ordre et leurs "frères les travailleurs ", comme si la pacification des relations entre les hommes était perçue comme un préalable nécessaire à leur propre émancipation.

Radiographie de la France démocratique

14 Selon des voies multiples et complémentaires les pétitionnaires s'efforcent donc de rendre leur protestation audible auprès d'un pouvoir qui, nous le verrons, se refuse à les écouter. Ces centaines de milliers de signatures recueillies en quelques semaines nous fournissent également, en creux, un témoignage précieux sur la répartition de l'opinion démocratique dans la France de la réaction conservatrice ${ }^{46}$. Le succès du pétitionnement s'explique par de multiples facteurs : il renvoie à l'état des forces du courant démocrate socialiste, à l'implantation et à l'organisation locale du parti républicain, ainsi qu'à l'ampleur de la surveillance policière et du contrôle social. La prise en compte de cette géographie pétitionnaire éclaire certains traits de la protestation, même si celle-ci est avant tout diverse et ne se laisse que difficilement réduire à des modèles explicatifs trop rigides.

15 En premier lieu, il semble que la géographie du pétitionnement recoupe d'assez près la répartition nationale des opinions avancées telle qu'elle apparaît lors des élections législatives du 13 juin $1849^{47}$. C'est bien la France démocrate qui pétitionne. Ainsi Paris, 
les régions situées au nord et à l'ouest du massif central, les départements alsaciens, méditerranéens et pyrénéens forment-ils les principales zones du pétitionnement ${ }^{48}$. La réussite du mouvement dépend donc étroitement du niveau d'implantation des montagnards. L'exemple des Pyrénées-Orientales est caractéristique à cet égard : 19 des 28 communes du département possédant un club politique actif en 1848-1849 sont également les principaux centres de collecte des signatures en mai $1850^{49}$. Parallèlement, les zones de faible vote démocrate sont le plus souvent peu actives dans le pétitionnement. Le Nord-Est (Ardennes, Moselle, Meuse), le bassin parisien et les départements situés au sud et à l'ouest de Paris (Seine-et-Oise, Eure-et-Loire, Loiret), et les pays de l'Ouest en général sont ainsi les moins actifs. Ces régions correspondent aux différentes implantations des conservatismes en France: l'Ouest armoricain, plutôt légitimiste, les provinces bourgeoises qui entourent Paris, de la basse Normandie à la Picardie et à la Champagne, d'orientation orléaniste ${ }^{50}$.

corrélation générale. La faiblesse du pétitionnement dans l'Allier, la Saône-et-Loire, l'Ain, et la faiblesse relative du pétitionnement du Rhône malgré le poids de Lyon, peuvent s'expliquer par la mise en état de siège de ces départements après les événements du 13 juin $1849^{51}$. De même, la faiblesse du pétitionnement dans la Haute-Vienne, et en particulier à Limoges, où les montagnards avaient pourtant remporté un large succès en 1849, correspond à la spécificité de l'implantation républicaine dans ce département ${ }^{52}$. Dès 1850 , la compression exercée par l'administration, mais également la reprise économique et l'attachement des ruraux au prince président, font retomber l'enthousiasme suscité en 1849 par l'élection des démocrates-socialistes ${ }^{53}$. La gauche limousine, malgré ses succès électoraux, est fragile, la politisation à gauche ne sera véritablement acquise qu'avec la Troisième République. La faiblesse du pétitionnement rend compte de cette fragilité et témoigne, avant même le coup d'État de décembre 1851 contre lequel la population protesta peu, des lacunes de l'implantation montagnarde. À l'inverse, l'Ouest aquitain (environ 9500 signatures sont collectées en Gironde) a pétitionné de manière importante alors que l'implantation montagnarde y était médiocre.

On peut peut-être y voir un phénomène d'acculturation à la pratique pétitionnaire lié aux grandes campagnes précédentes contre l'impôt des boissons. Un habitus pétitionnaire a pu se constituer à cette occasion dans les départements vinicoles. L'insertion dans des réseaux pétitionnaires antérieurs peut contribuer à expliquer la rapidité des formes de la mobilisation politique ${ }^{54}$.

Ces corrélations restent trop générales pour tenter une véritable anthropologie différenciée des pratiques pétitionnaires. Il faudrait insister davantage sur l'infinie diversité des facteurs explicatifs, l'influence des relations sociales locales comme les divers types de sociabilité. On peut néanmoins tenter d'élaborer une rapide typologie des formes de la mobilisation pétitionnaire en distinguant pétitionnements urbains et ruraux. Dans les départements à forte polarisation urbaine, les campagnes semblent marquées par l'inertie politique et l'opinion républicaine reste cantonnée dans les villes où l'influence des notables s'amenuise ${ }^{55}$. Dans le Rhône, 87 \% des signatures collectées l'ont été à Lyon. Dans les Bouches-du-Rhône, la Haute-Garonne ou le Doubs, près de la moitié des signatures sont recueillies respectivement à Marseille, Toulouse ou Besançon ${ }^{56}$. Cette inertie rurale a plusieurs explications : d'abord c'est en ville que l'on trouve les plus fortes implantations républicaines et la concentration démographique rend plus aisée la collecte des signatures malgré la surveillance étroite des autorités. L'inertie du 
pétitionnement rural peut témoigner également du conservatisme de certaines paysanneries : dans le Doubs, à la différence du Jura voisin, les campagnes ne sont pas gagnées "par la fièvre montagnarde ${ }^{57}$. Dans ce département, la loi réduit le nombre d'électeurs de $21 \%$, ce qui est peu par rapport à la moyenne nationale (30\%), et ce sont surtout les populations urbaines qui sont frappées (le nombre d'électeurs chute ainsi de $60 \%$ à Besançon et de $51 \%$ à Montbéliard) ${ }^{58}$. Dans ce département, ce sont les militants républicains ouvriers, principales victimes de la loi, qui se mobilisent. Les paysans restent, quant à eux, passifs. Comme l'explique le procureur général dans son rapport, les campagnes restent calmes, les paysans sont surtout soucieux des travaux des champs ${ }^{59}$.

Ailleurs, en revanche, le poids des campagnes et des signatures rurales devient prépondérant et explique largement le succès de la mobilisation. Ce second modèle est particulièrement bien illustré par le Gard étudié en détail par Raymond Huard ${ }^{60}$. Dans ce département, le troisième plus actif de France après Paris et le Nord, les pétitions recueillent plus de 15000 signatures collectées dans une centaine de communes. Les secteurs ruraux, pourtant moins touchés par la loi, sont les plus dynamiques. À Nîmes, où plus de 9800 électeurs sont privés du droit de vote, on recense à peine 1000 pétitionnaires, alors que dans le canton de Sommières par exemple, où 760 personnes sont privées du droit de vote, 1400 signatures sont récoltées. L'auteur en conclut que « la pétition n'est pas un simple réflexe de défense des catégories touchées par la loi, c'est un acte politique ${ }^{61}$. De nombreux départements actifs dans le pétitionnement répondent à ce modèle gardois. Dans la Sarthe, 11800 signatures sont récoltées dans 90 communes, dont seulement $16 \%$ au Mans. Dans le Bas-Rhin également, seuls $22 \%$ des 12252 signatures ont été recueillies à Strasbourg ${ }^{62}$. Dans ces départements, le pétitionnement se caractérise par la prépondérance de l'initiative villageoise et la dissémination rurale. Le pétitionnement permet alors d'apprécier l'organisation et la capacité d'action du parti républicain au niveau local. On entrevoit déjà certains des traits caractéristiques de l'insurrection de décembre 1851. Face au contrôle policier croissant qui s'exerce sur les villes, l'activité montagnarde se replie dans les campagnes.

Deux modèles se dessinent donc : celui des départements où la campagne joue un rôle de premier plan dans le pétitionnement - et dans ce cas on peut généraliser les conclusions de Raymond Huard; dans d'autres départements au contraire, ceux où les campagnes restent imperméables à toute influence des démocrates-socialistes (Nord et Doubs par exemple), la ville conserve une fonction mobilisatrice essentielle. Mais cette distinction doit elle-même être nuancée : dans certains cas, un pétitionnement rural autonome mobilisant des paysans non concernés par la loi côtoie un pétitionnement urbain ouvrier (comme dans la Haute-Garonne ${ }^{63}$ ).

Géographie du pétitionnement contre 


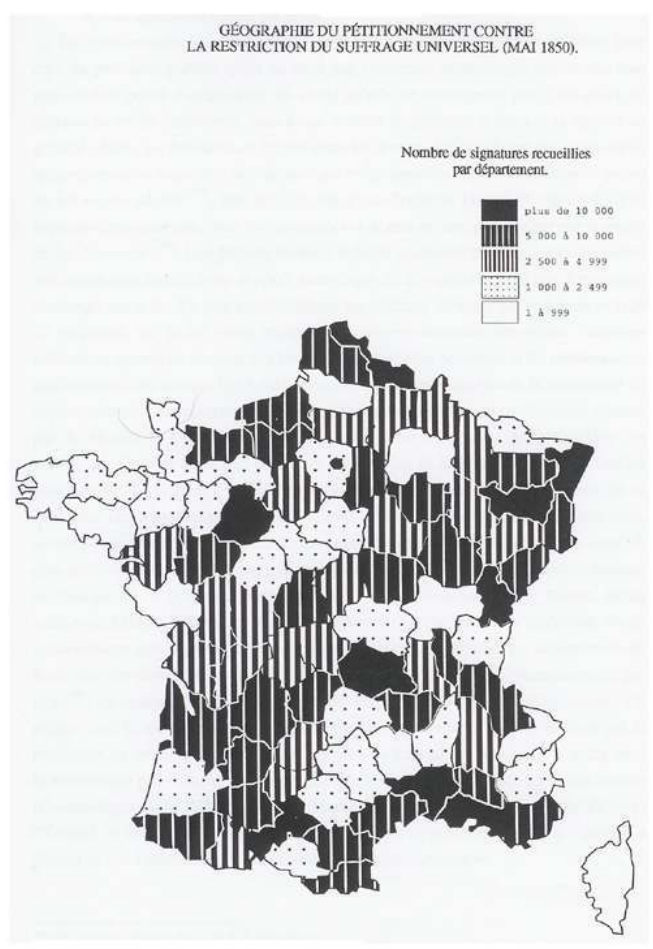

Du village au Parlement : les pétitions et l'expérience politique républicaine

Si les pétitions semblent révélatrices de la répartition complexe des opinions démocratiques, elles le sont également des modalités spécifiques du fonctionnement de la vie politique. En suivant le cheminement des pétitions et leurs usages différenciés, depuis leur collecte dans les villages et les quartiers jusqu'aux débats à l'Assemblée, on peut entrevoir certaines singularités de l'expérience politique républicaine. L'analyse classique du pétitionnement se limite souvent à l'étude des fonctions que lui assignent ceux qui en ont pris l'initiative; cela conduit à minorer l'impact réel des pétitions en limitant l'analyse à l'arène politique nationale. Pourtant, à chaque étape du pétitionnement, les acteurs y réinvestissent de multiples significations. Localement, la pétition peut être détournée de ses objectifs initiaux et instrumentalisée dans des luttes de clans ou des enjeux autres. Intégrant les analyses de Michel de Certeau sur « les arts de faire », l'étude des pétitions doit donc être attentive au "polythéisme» de ses usages disséminés ${ }^{64}$. Ainsi, la signification d'une pétition n'est pas fixée a priori au moment de sa rédaction, elle se construit progressivement dans la confrontation quotidienne des multiples arènes politiques décentralisées.

Par ces usages et appropriations diverses, la diffusion des pétitions, et à travers elles de mots d'ordre et de discours, contribue d'ailleurs au processus d'acculturation politique mis en lumière par l'historiographie. L'apprentissage de la politique n'est pas la conséquence d'un phénomène unique, comme l'introduction du suffrage universel. Ainsi que le note Gilles Pécout: «l'approche de la politisation est nécessairement reconstructive, ce qui lui donne son caractère de prime abord cumulatif [...], rien n'est explicable en dehors d'un faisceau de causes multiples : la structure du peuplement, les conditions d'intégration au marché économique national, les relations au sein du monde paysan, les rapports avec les autres catégories socioprofessionnelles, le degré 
d'instruction, tout cela influe globalement sur le mode d'entrée en politique des ruraux » ${ }^{65}$. En diffusant les grands thèmes de l'idéologie montagnarde (le recours au droit et à la constitution notamment), les pétitions participent indéniablement au mouvement de "descente de la politique vers les masses ${ }^{66}$.

Mais ce constat est insuffisant. La collecte des signatures constitue également un moment de dramatisation des enjeux politiques au cours duquel les représentations des acteurs se cristallisent. Dans le contexte politique troublé de la Seconde République, la signature d'une pétition n'est pas un acte anodin; elle peut entraîner des tumultes ou des attroupements inquiétants pour les autorités soucieuses d'ordre. Certains refusent d'ailleurs de la signer. À Montbéliard, sur la sollicitation d'une équipe de collecteurs de pétitions quadrillant la ville pour réunir les signatures, « M. Tuety, pasteur protestant, répond que sa position de pasteur lui interdit de s'occuper de politique et de signer une semblable pétition " ${ }^{67}$. Dans certains cas, des conflits réels avec les autorités locales ou entre clans opposés au sein de la communauté pouvaient surgir. Alors que de nombreux maires républicains acceptent de signer les pétitions en les rehaussant ainsi du maigre poids de leur légitimité ${ }^{68}$, d'autres, fidèles auxiliaires du pouvoir, s'efforcent au contraire de les interdire. À Châtenois, dans les Vosges, « 240 habitants avaient signé une première pétition que M. le Maire s'est permis de faire saisir et de brûler, puis appelant à son aide le juge de paix il espérait faire subir le même sort à la présente pétition, quand on s'est avisé de l'adresser à Paris, c'est pourquoi elle ne porte que 80 signatures " précise un collecteur scrupuleux ${ }^{69}$. Un autre pétitionnaire écrit au rédacteur du Travailleur de l'Indre pour dénoncer l'attitude du maire et du garde champêtre qui, «le 19 mai [...] ont eu pris les noms des électeurs voulant entreprendre de signer, ça a fait peur aux électeurs et les a empêché de signer la pétition ${ }^{70}$. Il semble que la collecte des pétitions entraîne un mouvement de déstructuration (division au sein du village) suivie d'une restructuration (identification à un clan) des relations sociales intra-communautaires, proche de ce que Christine Guionnet a observé à l'occasion des élections municipales de la Monarchie de Juillet $^{71}$. Les pétitions réactivent, en dehors de tout contexte électoral, les luttes partisanes au sein de la communauté. En entretenant ainsi les divisions, elles favorisent "l'avènement de la question identitaire », qui constitue une étape majeure du processus de modernisation politique ${ }^{72}$.

Une fois signée, la pétition rejoint la masse de celles qui s'entassent dans les bureaux de l'Assemblée. Ici, une seconde vie commence pour ces textes qui deviennent des armes dans les joutes oratoires auxquelles se livrent les représentants de la Montagne et du Parti de l'ordre. Pour les premiers, très minoritaires, les pétitions servent en quelque sorte de béquille de l'argumentation. En montrant, et en exagérant, la réprobation du pays, les représentants de la Montagne tentent de reconquérir une majorité symbolique. Le thème de "l'opinion publique » envahit leurs discours; en se présentant comme les porte-parole de cette opinion indéfinissable ils cherchent à capter un peu de sa légitimité. Pendant plusieurs jours, de longs défilés ritualisés de représentants de la Montagne apportent à la tribune des pétitions qui sont l'occasion de condamner le projet de loi. Pour Théodore Bac, à travers les pétitions, c'est «la conscience du pays qui parle » ${ }^{73}$. De son côté, la majorité conservatrice fait également un usage intensif de ce pétitionnement. Comme le résume sèchement Léon Faucher: «en quoi consiste le droit de pétition messieurs ? C'est le droit de supplique, c'est le droit de représentation » ${ }^{74}$. La pétition ne saurait être l'expression d'une opinion de la part d'un peuple «à jamais idiot et mineur " mais tout au plus celle d'une doléance humble et individuelle. Pour les élites 
traditionnelles, loin d'être l'expression d'une " opinion publique », le pétitionnement est plutôt un symptôme de l'anarchie qui trouble le pays et impose la réforme électorale.

D’ailleurs, la majorité conservatrice engage une véritable guerre aux pétitions, ne se contentant plus de l'ironie méprisante qui accompagnait souvent leur présentation sous la Monarchie de Juillet. Dans son rapport du $1^{\mathrm{er}}$ juin, Léon Faucher lance l'offensive en dénonçant le langage "grossier, insolent et séditieux " des pétitionnaires. Contre les 527000 signatures accusées d'être issues de la fraude, Faucher met en avant le contre pétitionnement conservateur organisé pour soutenir le projet de loi ${ }^{75}$. Dans les semaines qui suivent, la répression s'organise et 158 personnes sont poursuivies pour leur action lors de la collecte ${ }^{76}$. Ces poursuites, qui reproduisent la mécanique générale du contrôle policier sous la Seconde République, touchent surtout la moitié nord du pays. Les départements limitrophes de la capitale et les zones de contact entre Paris et les exilés belges sont au cœur de la répression. Outre ces poursuites, une quinzaine d'imprimeurs et gérants de journaux sont condamnés pour avoir omis de préciser leur nom sur les textes. À Paris, par exemple, Plon, l'imprimeur de La Presse, est condamné à une amende de 3000 francs. Dans les départements, les lourdes amendes obligent souvent les organes républicains à cesser de paraître. Ainsi, la répression contre le pétitionnement devient un moyen détourné pour supprimer quelques organes de l'opinion démocratique ${ }^{77}$. Au Parlement, comme au niveau local, chaque camp instrumentalise donc les pétitions selon ses propres objectifs politiques.

L'année suivante, de février à juillet-août 1851, une nouvelle campagne de pétitions est organisée, principalement dans le Midi, pour demander, cette fois, l'abrogation de la loi du 31 mai et, dans le même temps s'opposer à la demande de révision de la Constitution orchestrée dans tout le pays par les bonapartistes ${ }^{78}$. Mais cette seconde poussée pétitionnaire républicaine n'atteint pas l'ampleur de celle de mai 1850 . Comme le note Maurice Agulhon, « répression et découragement avaient porté quelques fruits depuis un an ${ }^{79}$. À l'image des barricades de Juin réprimées dans le sang, la barricade des pétitions échoue donc à bloquer l'évolution conservatrice du régime. Mais, comme on l'a souligné, la signification de ce pétitionnement ne se réduit pas à la seule question de la défense du suffrage universel masculin. Il s'inscrit plus globalement dans une série de mutations fondamentales des rapports au politique au milieu du XIX ${ }^{\mathrm{e}}$ siècle. Ce pétitionnement constitue en effet une étape importante dans la généalogie des formes de mobilisation politique. Par son ampleur, il représente l'une des premières campagnes de pétitions de masse redevable d'un espace public national unifié. Par ailleurs, comme l'a montré récemment Louis Hincker, les journées révolutionnaires de 1848 se sont accompagnées d'un apprentissage de "l'illégitimité du citoyen combattant» ${ }^{80}$. Face à cette délégitimation de la culture des armes, l'exercice de la citoyenneté se replie dans les formes pacifiées de l'écrit. Au moyen des pétitions les démocrates s'efforcent de reconquérir la légitimité perdue sur les barricades. 


\section{NOTES}

1.. Raymond HUARD, Le suffrage universel en France (1848-1946), Paris, Éditions Aubier, 1991, pp. 52-53.

2.. Karl MARX, Le 18 Brumaire de Louis Bonaparte, Paris, Éditions Messidor, 1984, p. 130.

3.. Robert BALLAND, « De l'organisation à la restriction du suffrage universel en France (1848-1850) », dans Jacques DROZ [dir.], Réaction et Suffrage universel en France et en Allemagne (1848-1850), Bibliothèque de la Révolution de 1848, tome XXII, 1963, pp. 114-142. 4.. Raymond HUARD, ouv. cité, p. 55.

5.. Maurice AGULHON, 1848 ou l'apprentissage de la République, Paris, Éditions du Seuil, 1992, p. 169.

6.. Dans une mise au point historiographique récente, Raymond HUARD invitait d'ailleurs à ouvrir ce chantier : « Le "Suffrage universel" sous la Seconde République. État des travaux, questions en attente ", dans Revue d'histoire du XIX $X^{e}$ siècle, 1997/1, n 14, p. 64. L'évocation de cette campagne de pétition est marquée par un certain impressionnisme. Ainsi, John Merriman, dans sa remarquable étude sur la Seconde République note que 78 départements pétitionnent (alors que nous avons trouvé des pétitions émanant de l'ensemble des départements, y compris de certaines colonies) et que le Doubs envoie plus de pétitions que le Jura voisin (alors que nous avons recensé 10600 signatures sur 124 pétitions dans le Jura contre seulement 6400 sur 150 pétitions dans le Doubs, mais le nombre de pétitions n'est pas un bon indicateur de l'ampleur de la mobilisation) ; voir John MERRIMAN, The Agony of the Republic. The Repression of the Left in Revolutionary France, 1848-1851, New Haven/Londres, Yale University Press, 1978, pp. 136-137.

7.. Dans les années 1840 en Grande-Bretagne les pétitions constituent une des principales formes de mobilisation politique, les vastes pétitionnements chartistes notamment réunissaient plusieurs millions de signatures, voir Paul A. PICKERING, «"And Your petitioners and C". Chartist petitionning in popular politics, 1838-1848 ", dans English Historical Review, 2001, 116 (466), pp. 368-388. À la même époque, les campagnes d'opinion françaises restent à des niveaux plus modestes : la célèbre « pétition de la garde nationale » recueille 150000 signatures en 1838-39 et 188000 en 1840, voir A. GOURVITCH, « Le mouvement pour la réforme électorale (1838-1841) », dans Bulletin de la société d'histoire de la révolution de 1848, tome XII, 1914-1916, pp. 93-131. Les pétitionnements anti-fiscaux sont sans doute ceux qui recueillent le plus d'adhésions, ainsi la campagne contre l'impôt des boissons en 1849 réunit environ 240000 signatures. 8.. Jean-Gabriel CONTAMIN, Contribution à une sociologie des usages pluriels des formes de mobilisation : l'exemple de la pétition en France, Thèse de doctorat en science politique sous la direction de Michel OFFERLÉ, Université de Paris 1, 2001, 816 p. Contre toute substantialisation des modes d'action politique, l'auteur propose d'étudier la pluralité des usages des pétitions. Au lieu de penser l'action pétitionnaire à partir de ses fonctions, de ce à quoi sert la pétition, il suggère de s'interroger « sur la manière dont les agents sociaux qui y sont pour une raison ou une autre intéressés s'en servent - et, de ce fait, la servent - de manières extrêmement diverses » (p. 159).

9.. À propos de l'attitude des montagnards, voir Maurice AGULHON, 1848 ou l'apprentissage de la République, ouv. cité,, p. 169. Les proscrits de Londres, dont Ledru-Rollin, sont les 
principaux défenseurs de la réponse insurrectionnelle, voir Pierre JOIGNAUX, Souvenirs historiques, Paris, Librairie Flammarion, 1891, volume 2, pp. 54-57. Cette attitude face au pétitionnement annonce la scission progressive qui s'opère entre la « Montagne parlementaire » majoritaire et attachée à la stratégie légaliste, et « la nouvelle Montagne ", encouragée par les exilés de Londres, qui privilégie la coordination des sociétés secrètes en vue de préparer un coup d'État, voir Inès MURAT, La Deuxième République, Paris, Librairie Arthème Fayard, 1987, pp. 433-434.

10.. Karl MARX, Les luttes de classes en France (1848-1850), Paris, Éditions Sociales, 1967, p. 161.

11.. Expression de Maurice TOURNIER dans « Le mot peuple en 1848, désignant social ou instrument politique », Romantisme, $n^{\circ}$ 9, 1975.

12.. Arch. nat. (Archives nationales), Pétitions adressées à l'Assemblée législatives. 1849-1851, C 2312.

13.. Publiée simultanément dans les trois journaux le 14 mai, cette « pétition de la réunion Goudchaux » est le texte le plus largement repris par la presse modérée de province.

14.. Ancien ministre de l'intérieur et rapporteur de la loi Baroche à l'Assemblée, ardent opposant aux démoc-soc, c'est lui qui introduit la législation de janvier 1849 interdisant les réunions politiques publiques, c'est également lui qui fait voter la loi du 16 juin 1850 établissant le timbre et le cautionnement pour les journaux, voir Marcel PAPAUD, « La répression durant le ministère de Léon Faucher (janvier-mai 1849) », dans Philippe VIGIER et Alain FAURE [dir.], Maintien de l'ordre et police en France et en Europe au XIXe siècle, Paris, Éditions Créaphis, 1987, pp. 87-102.

15.. Le Moniteur Universel, 2 juin 1850.

16.. Voir Maurice AGULHON, « Classe ouvrière et sociabilité avant 1848 », dans Histoire vagabonde, Paris, Éditions Gallimard, 1988, tome 1, pp. 60-97 ; Jacques ROUGERIE, « Le mouvement associatif populaire comme facteur d'acculturation politique à Paris de la Révolution aux années 1840. Continuités et discontinuités ", dans Annales historiques de la Révolution française, 1994, $\mathrm{n}^{\circ} 3$, pp. 493-516.

17.. Arch. nat., BB18 385-390, rapport du procureur général du parquet d'Amiens au garde des sceaux, 25 juin 1851.

18.. Arch. nat., C 2312 : à Neuve-de-Liard (Puy-de-Dôme), les signatures sont recueillies devant l'église à la sortie de la messe. Comme le rappelle John Merriman, à Paris les étudiants font circuler des pétitions dans le cours de Michelet au collège de France ; voir The Agony of the Republic, ouv. cité, pp. 67 et 100.

19.. À Arlay (Jura) on précise ainsi que « si cette pétition n'est pas propre, c'est qu'elle a été signée en grande partie à travers les champs et les vignes, où se trouvaient nos vignerons et cultivateurs ", Arch. nat., C 2303.

20.. La Presse, 15 mai 1850. «Quelques tables dispersées à l'entrée de La Presse, de grandes feuilles de papier, quelques employés pour contenir la file d'attente, diriger les protestataires... Girardin observe de son étage le succès de son initiative : déjà deux mille..., déjà quatre mille signatures... Dix mille Parisiens viendront le premier jour » précise Paul PELISSIER, dans Émile de Girardin, prince de la presse, Paris, Éditions Denoël, 1985, pp. 265-266.

21.. Alors que sous la Monarchie de Juillet la mention du statut du signataire constituait une dimension prépondérante de l'usage des pétitions, en mai 1850 on assiste au contraire au développement de l'anonymat, ce qui rend d'ailleurs problématique toute comptabilité précise du nombre d'adhésions à la pétition. Dans certains départements, les 
pétitions sont couvertes de croix suivies du nom du signataire inscrit par le collecteur de pétition. Dans d'autres zones, ceux qui ne savent pas signer sont exclus de la protestation : à Limard (Haute-Vienne), les 420 électeurs de la commune déclarent tous adhérer à la pétition, mais seul une quarantaine d'entre eux ont signé, les autres « n'ont pu signer la pétition ne sachant ni lire ni écrire ", Arch. nat., C 2309.

22.. Le Démocrate du Rhin, 18 mai 1850.

23.. Ces analyses portent sur les 40 textes de pétition ( 9000 mots) les plus fréquents et les plus largement répandus dans le pays.

24.. Sur l'importance du mot « peuple » comme indicateur de la structure du discours politique de la Seconde République, voir Maurice TOURNIER, « Le peuple quarantehuitard : un outil de classe », dans Propos d'étymologie sociale, Paris, Éditions Klincksiek, 1992, volume 1, pp. 93-133, et art. cité.

25.. Ostwald DUCROT, «L'argumentation par autorité », dans Le dire et le dit, Paris, Éditions de Minuit, 1984, p. 158 et suiv.

26.. Arch. nat., C 2300, pétition de Saint Laurent (Ardennes).

27.. Arch. nat., C 2312, texte imprimé de la pétition diffusée en Dordogne.

28.. Pour désigner la commission, composée de 17 membres, chargée de présenter un rapport sur le projet de loi Baroche.

29.. Arch. nat., C 2302, Saint-Ambroise (Gard).

30.. Une pétition des Bouches-du-Rhône va jusqu'à qualifier le projet de loi de « tentative anarchique ", poussant jusqu'au bout l'instrumentalisation des mots de la réaction.

31.. Albert. O. HIRSCHMAN, The Rhetoric of Reaction : Perfersity, Futility, Jeopardy, Harvard, Harvard University Press, 1991, traduction française Deux siècles de rhétorique réactionnaire, Paris, Librairie Arthème Fayard, 1991, p. 169 et suiv.

32.. Cité par Pierre ROSANVALLON, Le sacre du citoyen, histoire du suffrage universel, Paris, Éditions Gallimard, 1992, p. 305.

33.. Comme l'écrit Pierre ROSANVALLON, « l'idée que l'inclusion de tous dans la vie politique grâce à l'élargissement du droit de vote va supprimer les ferments révolutionnaires est alors largement partagée », idem, p. 290.

34.. Arch. nat., C 2303, Morbihan.

35.. Pierre ROSANVALLON, Le sacre du citoyen, histoire du suffrage universel en France, ouv. cité., pp. 169-170.

36.. Sur ce point voir Odile KRAKOVITCH, « Les pétitions seul moyen d'expression laissé aux femmes, l'exemple de la Restauration », et Michèle RIOT-SARCEY, « Les femmes protestent sous la monarchie de Juillet », dans Alain CORBIN, Jacqueline LALOUETTE et Michèle RIOT-SARCEY [dir.], Femmes dans la cité, Paris, Éditions Créaphis, 1998, pp. 347-371et pp. 389-400.

37.. Principalement à Paris où 700 signatures sont recueillies sur 44 pétitions, l'une d'entre elles émane de « l'association fraternelle des ouvrières de lingerie » et porte la signature de Jeanne Deroin (Arch. nat., C 2306). On en trouve également à Gonfaron (Var) et Châtellerault (Indre et Loire) où deux pétitions de femmes sont signées à côté des pétitions masculines (Arch. nat., C 2309 et 2312). Pour éviter l'accusation de fraude promptement mise en avant par les autorités pour invalider les pétitions, les femmes évitent de mêler leurs signatures à celles des hommes.

38.. Arch. nat., C 2306, pétition des « femmes démocrates » (Paris).

39.. Idem.

40.. Sur les relations entre les femmes et le saint-simonisme, voir par exemple Christiane PLANTÉ, « Les féministes saint-simoniennes, possibilités et limites d'un mouvement 
féministe en France au lendemain de 1830 », dans Jean-René DERRÉ [dir.] Regards sur le saint-simonisme et les saint-simoniens, Lyon, Presses Universitaires de Lyon, 1986, p. 78. 41.. Voir Geneviève FRAISSE, « Les femmes libres de 1848, féminisme et moralisme », Les Révoltes logiques, $\mathrm{n}^{\circ} 1,1975$, repris dans Geneviève FRAISSE, Les femmes et leur histoire, Paris, Éditions Gallimard, 1998, pp. 352-355.

42.. Idem, p. 322.

43.. Idem, p. 318.

44.. Arch. nat., C 2306, pétition imprimée diffusée à Paris.

45.. Voir Michèle RIOT-SARCEY, La démocratie à l'épreuve des femmes. Trois figures critiques du pouvoir (1830-1848), Paris, Éditions Albin Michel, 1994, 365 p.

46.. Ces pétitions sont conservées aux Archives Nationales sous les cotes C 2300 à C 2314 . Léon Faucher, lors de son rapport au nom de la commission des pétitions le $1^{\mathrm{er}}$ juin 1850 , mentionne 527000 signatures (Le Moniteur Universel du 2 juin). Nos recensements nous donnent seulement 470000 signatures. On ne peut pas soupçonner Léon Faucher, qui soutient le projet de loi, d'avoir voulu grossir le poids du pétitionnement. Ce décalage peut provenir de la disparition de certaines pétitions. Le décompte des signatures à l'Assemblée ne pouvait être qu'approximatif étant donné le nombre massif de pétitions qui y affluaient. En outre, certaines ont donné lieu à des poursuites judiciaires et ont été renvoyées dans les différents parquets de cours d'appel où beaucoup ont pu se perdre. Raymond HUARD reprend le nombre avancé par Léon Faucher ; Maurice AGULHON se contente, avec raison, d'évoquer 500000 signatures, dans 1848 ou l'apprentissage de la république, ouv. cité, p. 169. Ce chiffre constitue une voie médiane raisonnable entre le nombre proposé par Léon Faucher et le nôtre.

47.. Jacques BOUILLON, « Les démocrates-socialistes aux élections de 1849 », dans Revue française de science politique, 1956, $\mathrm{n}^{\circ} 1$.

48.. Voir la carte ci-jointe. La même corrélation s'observe à l'échelle régionale. Dans les Alpes, par exemple, l'Isère et la Drôme pétitionnent davantage que les Hautes-Alpes qui connaissent une implantation républicaine plus fragile; voir Philippe VIGIER, La Seconde république dans la région alpine. Étude politique et sociale, Paris, Presses Universitaires de France, 1963, tome II, pp. 214-221.

49.. Les pétitions précisent rarement si elles proviennent d'un club politique, ce qui les desservirait auprès des autorités ; voir Peter McPHEE, Les Semailles de la République dans les Pyrénées-Orientales (1848-1852). Classes sociales, politique et culture, Perpignan, Publications de l’Olivier, 1995, pp. 457-458.

50.. Georges DUBY et Armand WALLON [dir.], Histoire de la France rurale, tome 3 : Apogée et crise de la civilisation paysanne de 1789 à 1914, Paris, Éditions du Seuil, 1976, pp. 156-157. 51.. Le Rhône n'arrive qu'en $18^{\text {ème }}$ position avec un peu plus de 7500 signatures (dont 6500 collectées à Lyon). Vincent ROBERT souligne qu'après la proclamation de l'état de siège les « rouges » utilisent les convois funéraires, « le seul type de cortège qui ne pouvait être poursuivi ", pour marquer leur emprise sur la population ; voir Les chemins de la manifestation (1848-1914), Lyon, Presses Universitaires de Lyon, 1996, p. 111. On peut ajouter que la collecte des signatures constituait une autre occasion propice à la démonstration de l'influence républicaine dans la ville.

52.. Alain CORBIN, Archaïsme et modernité en Limousin, Paris, Éditions Marcel Rivière, 1975, pp. 743-754. 4700 signatures furent récoltées dans environ 25 communes, et seulement 500 signatures à Limoges.

53.. Idem, pp. 830-831, selon Alain Corbin, « il est révélateur de constater que lors de la révision des listes électorales effectuées au lendemain de la loi du 31 mai 1850, un grand 
nombre de paysans qui en avaient le droit ont négligé de se faire inscrire ». S'ils ont négligé de se faire inscrire sur les listes électorales, on peut comprendre qu'ils ne se soient pas mobilisés pour défendre le maintien du suffrage universel.

54.. Ce que tend à confirmer une microanalyse des pétitionnaires d'Esslingen montrant comment un mouvement pétitionnaire s'élargit progressivement dans le temps à partir d'un noyau initial de gens déjà habitués aux pratiques civiques : voir Carola LIPP et Lothar KREMPEL, « Petitions and the Social Context of Political Mobilization in the Revolution of 1848/49: A Microhistorical Actor Centered Network Analysis ", dans International Review of Social History, volume 46, supplément 9, 2001.

55.. Peter McPHEE, The Politics of the rural life. Political Mobilization in the French countryside (1846-1852), Oxford, Clarendon Press, 1992, pp. 148 et suiv.

56.. En Seine-inférieure, autre cas intéressant, près de 9400 signatures sont collectées (Arch. nat, C 2314). Le rôle prépondérant des militants démocrates-socialistes apparaît nettement dans la prépondérance du Havre (3 200 signatures recueillies dans l'arrondissement) sur Rouen (seulement 1000 signatures) ; voir Pierre ARDAILLOU, Les Républicains du Havre au XIXe siècle (1815-1889), Rouen, Publications de l'Université de Rouen et du Havre, 1999, pp. 118-119.

57.. Dans son étude de la " genèse d'une paysannerie conservatrice », Jean-Luc Mayaud montre les résistances de certains mondes ruraux face à l'influence des républicains avancés. Les administrateurs locaux reprennent très vite en main l'encadrement des populations rurales, et 1237 cabarets sur 2775 sont fermés dans le département ; JeanLuc MAYAUD, Les République du Doubs, Annales littéraires de l'Université de Besançon, Éditions les Belles lettres, 1986, pp. 295 et 310.

58.. Idem, pp. 310-311.

59.. Arch. nat., BB30 373, rapport du procureur général de la cour d'appel de Besançon, 28 juin 1850.

60.. Raymond HUARD, « La défense du suffrage universel sous la Seconde République, les réactions de l'opinion gardoise et le pétitionnement contre la loi du 31 mai 1850 (1850-1851) ", dans Annales du Midi, octobre-décembre 1971, pp. 315-336. Grâce à l'échelle d'observation départementale, l'auteur a pu se livrer à une fine analyse des conditions locales d'organisation du pétitionnement.

61.. Idem, pp. 325- 326.

62.. On pourrait évidemment multiplier les exemples (Puy-de-Dôme, Gironde, Pas-deCalais, Pyrénées-Orientales etc.) ; pour une présentation plus détaillée de ces données statistiques nous renvoyons à notre mémoire de maîtrise : L'attitude de l'opinion face à la réaction conservatrice..., mémoire cité, pp. 151 et suiv.

63.. Dans ce département il existe en effet un pétitionnement spécifiquement urbain (4 500 signatures recueillies à Toulouse sur des pétitions imprimées comme supplément du journal montagnard La Civilisation) et un autre qui semble émaner d'initiatives locales (environ 6000 signatures recueillies sur des pétitions manuscrites et provenant de nombreuses communes du département), Arch. nat., C 2302.

64.. Selon Jean-Gabriel Contamin, « la pratique pétitionnaire ne saurait être réduite aux usages qu'en font les autorités ». Les pétitions, et plus généralement, la prégnance du fait pétitionnaire, ne peut se comprendre qu'à l'aune des multiples appropriations dont elle est l'objet à chaque étape de sa formation; voir Jean-Gabriel CONTAMIN, Contribution à une sociologie des usages pluriels des formes de mobilisation, thèse citée, pp. 162-163. 65.. Gilles PÉCOUT, « La politisation des paysans au XIX ${ }^{e}$ siècle, réflexion sur l'histoire politique des campagnes françaises ", Histoire et sociétés rurales, n 2, 1994, p. 119. 
66.. Maurice AGULHON, La République au village. Les populations du Var de la Révolution à la Seconde République, Paris, Éditions du Seuil, 1979, chap. VI.

67. Arch. nat., BB30 334, Rapport du procureur général de la cour d'appel de Besançon au garde des sceaux, le 2 juin 1850.

68. Sur les 751 dissolutions prononcées par le conseil d'État du $1^{\mathrm{er}}$ avril 1849 au 28 avril 1851,55 le furent en raison de la participation au pétitionnement contre la loi Baroche en mai 1850, voir John M. MERRIMAN, The Agony of the Republic, ouv. cité, p. 116.

69.. Arch. nat., C 2310.

70.. Arch. nat., C 2311, Déols, Indre.

71.. Christine GUIONNET, L'apprentissage de la politique moderne, les élections municipales sous la monarchie de Juillet, Paris, Éditions de L'Harmattan, 1997.

72.. Idem, p. 295.

73. Théodore Bac est un membre actif de la Montagne, avocat spécialisé dans les procès de presse sous la Monarchie de Juillet, en 1848 il est nommé commissaire du gouvernement dans la Haute-Vienne, sous la Législative il s'oppose activement à tous les projets conservateurs, voir Adolphe ROBERT et Gaston COUGNY, Dictionnaire des parlementaires français, Paris, Bourloton Éditeur, 1891.

74.. Le Moniteur universel, 26 mai 1850, p. 4007.

75.. Pourtant, ce contre-pétitionnement ne réunit que 2548 signatures laborieusement collectées par quelques journaux et conservateurs locaux. $86 \%$ des signatures proviennent de deux départements : la Loire-Inférieure et la Dordogne ; ce fut largement un échec, voir Arch. nat., C 2393, n 1742, 1753.

76.. « Rapports sur les poursuites auxquelles ont donné lieu les pétitions contre le projet de loi du 31 mai 1850 », Arch. nat., BB18 1485 A.

77.. La presse constitue l'une des cibles principales de la politique de réaction : les attaques contre le président de la République sont interdites, tous les journaux politiques devaient imprimer les décrets du gouvernement, on rétablit le cautionnement, une copie de chaque édition devait être déposée à la préfecture avant sa vente, et, après juillet 1850, chaque article devait être signé ; voir John M. MERRIMAN, The Agony of the Republic, ouv. cité, pp. 30-31.

78. La Constitution interdisait au Président de se faire réélire pour un second mandat, pour contourner cet interdit Louis-Napoléon Bonaparte fait de la révision de la Constitution l'un de ses grands mots d'ordre. Pendant l'été 1850, les préfets invitent les conseils généraux à voter des vœux en ce sens puis, au cours du printemps 1851, le pouvoir bonapartiste relance la campagne révisionniste sous la forme d'un vaste mouvement pétitionnaire. C'est en réponse à cette offensive bonapartiste que les républicains du Midi recommencent le pétitionnement contre la loi du 31 mai 1850. 79.. Maurice AGULHON, 1848 ou l'apprentissage de la République, ouv. cité, p. 178.

80.. Louis HINCKER, Être insurgé et être citoyen à Paris durant la Seconde République, thèse de doctorat d'histoire sous la direction d'Alain CORBIN, Université Paris 1, 2003, voir notamment le chapitre 9 : «L'illégitimité de la culture des armes ». 


\section{RÉSUMÉS}

$\mathrm{Au}$ cours du processus de réaction conservatrice qui caractérise l'évolution de la Seconde République, le vote de la loi restreignant le suffrage universel masculin le 31 mai 1850 constitue un moment charnière. Devant la répression accrue, la résistance à cette loi prit essentiellement la forme d'un vaste mouvement de pétitionnement qui, en quelques semaines, parvint à réunir plus de 500000 signatures. À travers l'étude de ce pétitionnement exceptionnel, cet article envisage certains aspects de la mobilisation démocratique en France au milieu du XIX ${ }^{\mathrm{e}}$ siècle. Nous examinerons les modalités de cette campagne en suivant les stratégies de la collecte, la géographie de la France pétitionnaire et les usages parlementaires de ces pétitions.

A 'barricade of papers' : the petition movement against the restriction of male universal suffrage in May 1850.

During the reactionary conservative process which characterized the evolution of the Second Republic, the vote of the law restricting the male universal suffrage on 31 May, 1850 was a turning point. Facing the growing repression, the resistance to this law essentially took the form of a vast petition movement which, in only a few weeks, succeeded in collecting over 500,000 signatures. With the study of this exceptional petition movement, this article considers some aspects of democratic mobilization in mid-nineteenth- century France. It will examine the methods of this campaign by following the strategy used for the collection of these petitions, their geography and the parliamentary use of these petitions.

\section{AUTEUR}

\section{FRANÇOIS JARRIGE}

allocataire-moniteur en histoire contemporaine à l'Université Paris 1-Panthéon-Sorbonne (Centre de recherche en histoire du XIX ${ }^{\mathrm{e}}$ siècle) 